\title{
Phlebosclerotic colitis: radiological findings of an uncommon entity
}

\author{
HY Chan *, MN Chan, F Ng, WT Ho
}

Hong Kong Med J 2015;21:573.e1-2

DOI: 10.12809/hkmj154585

A 46-year-old Chinese woman attended our accident and emergency department because of abdominal pain, no bowel movements, and vomiting for 10 days in February 2014. A plain abdominal radiograph revealed a dilated small bowel, ascending and transverse colon (Fig 1). Multiple short linear

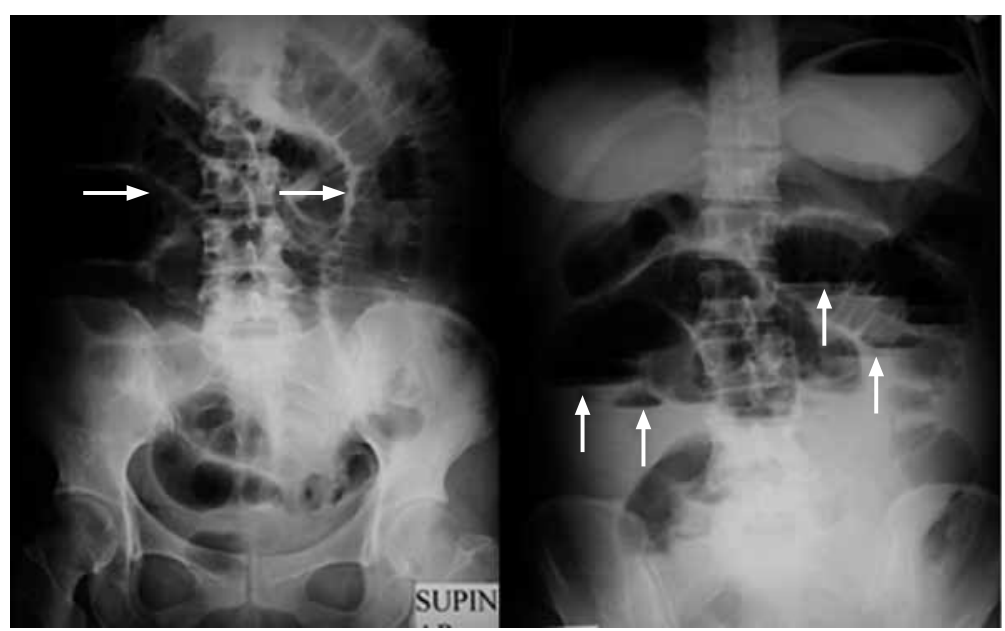

FIG I. Abdominal radiographs showing dilated small bowel, ascending and transverse colon with multiple air-fluid levels (arrows)

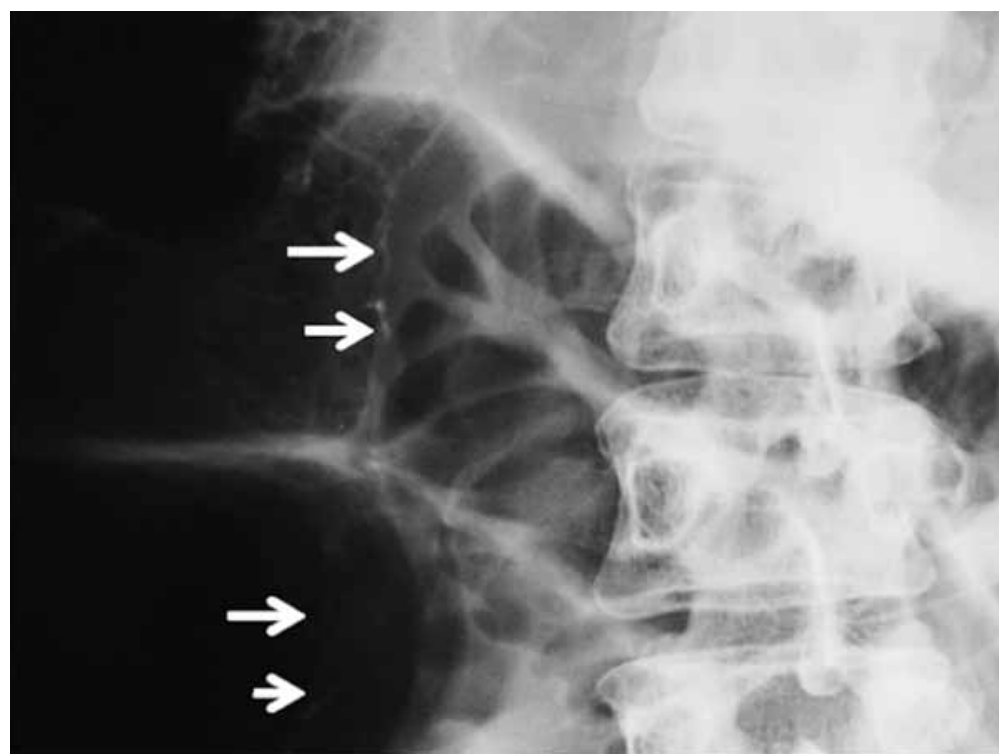

FIG 2. An abdominal radiograph revealing multiple short linear calcifications along the medial aspect of ascending colon (arrows) calcifications scattered along the medial aspect of the ascending colon were observed (Fig 2). Contrastenhanced computed tomography $(\mathrm{CT})$ revealed a long segment of circumferential bowel wall thickening and oedema involving the caecum, the whole length of the ascending colon, hepatic flexure, and the proximal part of the transverse colon. Prominent vascular calcifications that involved the mesenteric vessels over the ascending colon were also visualised (Fig 3).

The patient was admitted to a surgical unit and emergency laparotomy was arranged with a provisional diagnosis of intestinal obstruction. Intraoperatively, the colon was found to be ischaemic from the caecum to the splenic flexure. The small bowel was dilated and the superior mesenteric artery was patent and pulsatile. Extended right hemicolectomy and end ileostomy were performed. Histopathology of a surgical specimen showed most of the submucosal veins to have luminal occlusion by intimal thickening and a hyalinised wall with calcification. The pathological diagnosis was phlebosclerotic colitis (PC). Postoperatively, the patient's recovery was complicated by pneumonia that was successfully treated with antibiotics. She was discharged home 18 days after admission. Elective closure of the end ileostomy was performed a few months later.

Ischaemic bowel disease is commonly caused by thrombosis and embolism in the mesenteric artery. Obstructed mesenteric veins causing ischaemia are rarely reported. Phlebosclerotic colitis is characterised by sclerosis and calcification of the mesenteric veins leading to large bowel ischaemia. Interestingly, a genetic factor is thought to play an important role in this disease since most patients with PC are of Asian descent. It has been suggested that portal hypertension may contribute to the condition but there is insufficient evidence to support this relationship. ${ }^{1}$

The most common symptoms of PC are abdominal pain, vomiting, and recurrent diarrhoea. The clinical course is fairly long because it is caused by chronic venous insufficiency and congestion. Serious complications including ileus and intestinal perforation have been reported. ${ }^{2,3}$ Phlebosclerotic colitis has distinct radiological findings. Plain radiographs may demonstrate multiple tortuous threadlike vascular calcifications commonly over the 


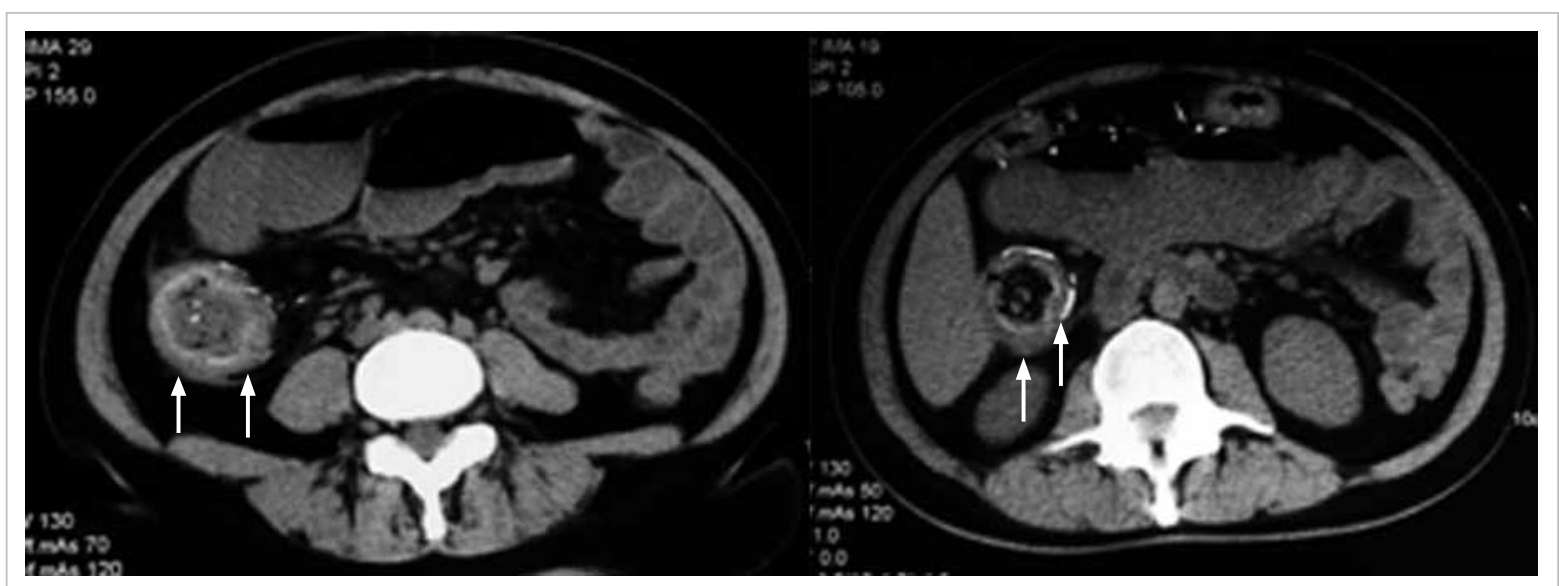

FIG 3. Abdominal computed tomographic scans demonstrating prominent vascular calcifications involving the mesenteric vessels over the ascending colon with thickening of the colon wall (arrows)

ascending colon and may extend to the transverse colon. Findings from CT include mucosal thickening with calcifications along the colonic wall and around the superior mesenteric vein trunk. Colonic wall thickening caused by oedema and fibrosis can be seen in more detail with magnetic resonance imaging although calcifications cannot be demonstrated. Endoscopic examination typically shows dark purple-blue mucosal change of the involved colon and oedema because of venous congestion. A rigid colon and ulceration are also observed.

There is no standardised treatment for PC. Many authors suggest that conservative management is sufficient unless the disease is severe or results in complications such as peritonitis and sepsis. ${ }^{4}$ Nonetheless most reported cases have been treated surgically. A few patients have obtained symptomatic relief following conservative treatment but serious relapse later has eventually resulted in a need for surgery. ${ }^{1}$

HY Chan *, FHKCEM, FHKAM (Emergency Medicine)
MN Chan, MB, BS

$\mathrm{F} \mathrm{Ng}$, FHKCEM, FHKAM (Emergency Medicine)

WT Ho, FHKCEM, FHKAM (Emergency Medicine)

Accident and Emergency Department, Caritas Medical Centre,

Shamshuipo, Hong Kong

* Corresponding author: chanhy1@ha.org.hk

\section{References}

1. Wang $\mathrm{CH}$, Chen TY, Chin J, Wu YJ, Wang MT. Phlebosclerotic colitis: a case with a history of herbal ingestion. J Soc Colon Rectal Surgeon (Taiwan) 2012;23:129-34.

2. Yao T, Iwashita A, Hoashi T, et al. Phlebosclerotic colitis: value of radiography in diagnosis-report of three cases. Radiology 2000;214:188-92.

3. Kato T, Miyazaki K, Nakamura T, Tan KY, Chiba T, Konishi F. Perforated phlebosclerotic colitis-description of a case and review of this condition. Colorectal Dis 2010;12:14951.

4. Yu CJ, Wang HH, Chou JW, et al. Phlebosclerotic colitis with nonsurgical treatment. Int J Colorectal Dis 2009;24:1241-2. 